\title{
A Specified Saliency Detection for Human Detection
}

\author{
Ling Zheng a,b*, Shuhan Chen ${ }^{\mathrm{b}}$, Xuelong $\mathrm{Hu}^{\mathrm{b}}$, Lifeng Zhang ${ }^{\mathrm{a}}$ \\ ${ }^{a}$ Kyushu Institute of Technology \\ 1-1, Sensui, Tobata, Kitakyushu, Japan 804-8550 \\ b Yangzhou University \\ 196, Huayangxilu, Yangzhou, Jiangsu, China 225-127 \\ *Corresponding Author: zlapgx@ 163.com
}

\begin{abstract}
Saliency detection algorithm plays an important role in computer vision tasks, such as object recognition, visual tracking, image compression, image segmentation and so on. Fortunately, it is also a useful technique for monitoring the status of aged automatically in a short time span. Saliency detection can detect the aged shapes directly and distinguish the giant pets easily. It can prevent the aged "lonely death", furthermore it is a private way for detecting which results are gray saliency maps that not contain any information of the aged except body shape. To addressed it, we present a simple and efficient bottom-up saliency detection model. In specially, we first propose a discriminative histogram feature metric by combing multiple color space and gradient magnitude channel to handle complex images. Then, we use this metric to compute background based coarse saliency map by introducing distribution as prior to remove foreground noises in the image boundaries. Finally, a simple refinement is applied to generate pixel-wise and smooth saliency maps. The experimental result shows the possibility that the proposed method is able to detect the active state of aged.
\end{abstract}

Keywords: Saliency detection, Computer vision,

Discriminative histogram

\section{Introduction}

With the development of aging of the Japan Population, the home alone elder "lonely death" situation has become a serious problem which draws us attention. According to Japanese government statistics, there are $1 / 4$ old male who will die lonely, and the reason is they are single and no one around to take care of them. They were found several days after their death. The Japanese government is seeking the solution to this social problem. Naturally, we need find a detect technology which is useful technique for monitoring the existence and active state of old person automatically in a short time span. Generally, face recognition technique has been expected to realize this task. However, it is difficult to detect the existence in every situation only by using the face recognition technique. For example, if a person backward to the camera or just a part of body appearing in the frame, the facial feature can't be obtained.

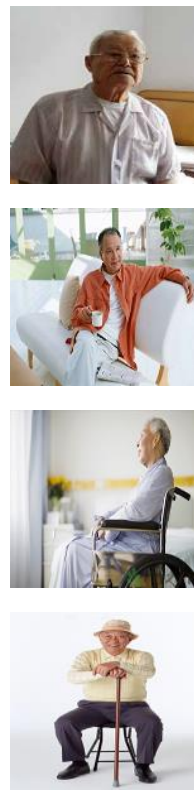

(A)
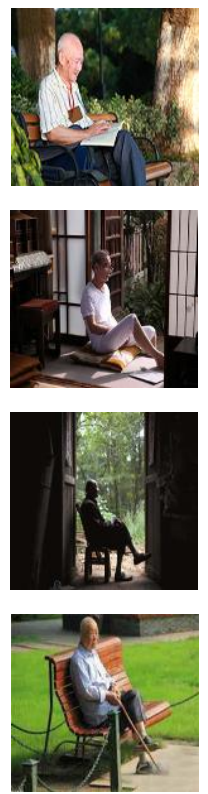

(B)
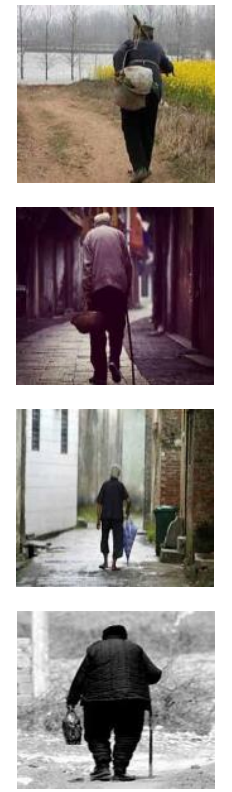

(C)
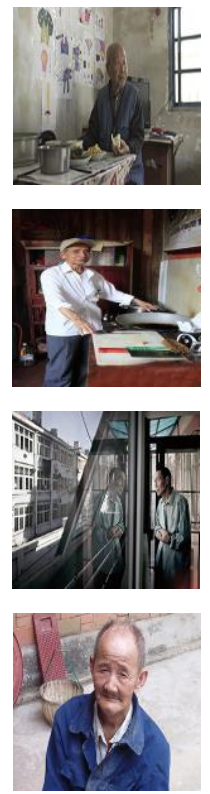

(D)
Fig.1. Experiment examples of aged pictures. Saliency detection is ambiguous and task dependent. Different background and different pose could be a influence factor. As we can see the (A) are more bright picture, the (B) are the sit pose and toward the same direction, the $(\mathrm{C})$ are the back view and the (D) are the front view.

Recent years have witnessed rapidly increasing interest in salient object detection [1]. Saliency detection aimed at 
detecting the most important part of an image has become a very active topic in both neuroscience and computer vision in recent years, which measures the low-level stimuli that grabs viewers' attention in the early stage of human vision [2], whose research mainly contains three aspects: eye fixation prediction [3], saliency detection [4], and objectness estimation [5]. In this paper, we focus on saliency detection which aims to make certain objects or regions of an image stand out from their neighbors and catch immediate attention. It is still challenging to develop efficient saliency detection algorithms which plays an important role in the wide range of computer vision tasks, such as object recognition [6], image retargeting [7], visual tracking [8], image compression [9] and so on.

Early works mainly concentrate on low-level cues, such as features, colors and spatial distances. One of the most used principles, background prior, is to take the contrast versus a narrow border of the image as a region's saliency. However, it will be imprecise and directly lead to undesirable results when the object is adjacent to one or more image boundaries. Contrast prior is another widely used principle by measuring the local or global contrast difference between the object regions with respect to other regions in the image to compute saliency.

Recently, saliency propagation based approaches have been proposed and achieved state-of-the-art performance. In detail, the input image is represented by a graph with segmented superpixels and edge strength proportional to superpixel similarity [10]. Saliency values are conducted by different propagation models diffused along these edges from labeled superpixels to unlabeled neighbors. Although several seed mechanisms have been proposed in the literature, they tend to be heuristic in nature or learning based [10]. However, they may cause errors when the foreground seeds are not accurate enough.

In this paper, we present a simple and efficient method to overcome the drawbacks of the previous saliency propagation mentioned above. Firstly, we use distribution prior to reduce the foreground noise which filter out the superpixels in the boundaries that most unlikely belong to the background so that obtain more stable and reliable background prior knowledge. Secondly, a discriminative feature metric is proposed as similarity measure combing three color spaces (CIE LAB, HSV) and gradient magnitude channel to handle complex images. It is worth to say this model which does not need iteration is more simple and efficient. Finally, we use sigmoid function and fast bilateral filter [11] to produce smooth and pixel-wise saliency maps.

The rest of the paper is organized as follows. Section 2 introduces our proposed method. Section 3 shows the experiment results. Finally, conclusions are drawn in Section 4.

\section{Proposed Method}

We present an effective and robust saliency detection frame work. At first, an input image is segmented into a fixed number of superpixels by the simple linear iterative clustering (SLIC) algorithm [12]. Then, we introduce our

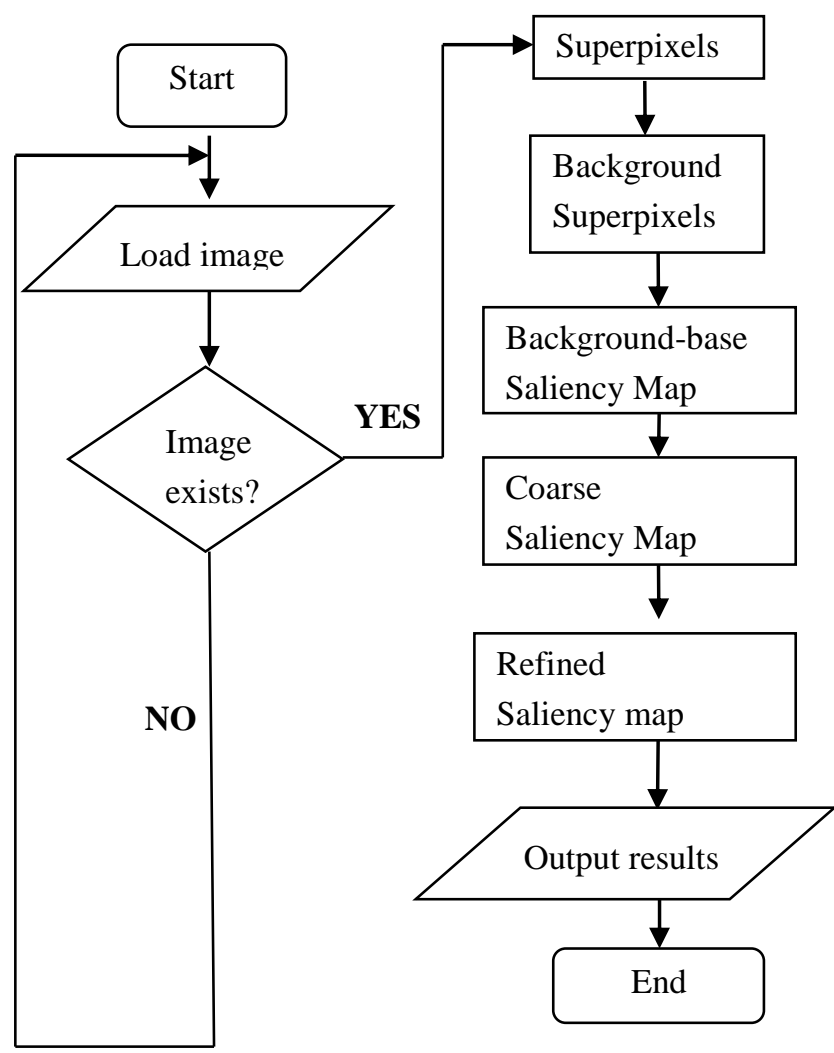

Fig.2. Diagram of proposed method

discriminative feature metric considering multiple color spaces and gradient magnitude to describe each superpixel, which can be applied both in coarse map generation and saliency propagation and make vital importance to them. After that, a background based weak saliency model is proposed by using distribution prior. Finally, we refine the generated saliency map by sigmoid function and fast bilateral filter. The pipeline of the propose algorithm is shown in Fig. 2.

\subsection{Distribution Guided Backgroundness}

Backgroundness is a very effective cue and has been widely used in saliency detection, which assumes the image 
borders as prior background regions. However, it will lead to negative effects on saliency detection due to some foreground noises in the border regions. Then, some works try to solve it by calculating distinctiveness only among image boundaries. Unfortunately, it will lose its effectiveness when the background is complex. In such case, some background regions may be distinctive and then be removed as foreground noises. Thus, we use the effective cue distribution of the whole image as prior to remove such foreground noises, which measures saliency by spatial variance. Low variance indicates more salient object than spatially widely distributed superpixels [15]. In detail, it is defined as below:

$$
D_{i}=\frac{1}{Z} \sum_{i}^{N} \exp \left(-\frac{h_{i j}}{2 \sigma_{d}^{2}}\right) \cdot\left\|p_{j}-\mu_{i}\right\|^{2}
$$

where $h_{i j}$ is the chi-square distance between two histograms of superpixel $i$ and $j$ in our multiple color spaces, $P_{j}$ is the position of superpixel $j, Z_{i}$ is the normalization term, $\mu_{i}$ defines the weighted mean position of superpixel $i$, and $N$ is the number of superpixels. The parameter $\sigma_{d}$ controls the color sensitivity of the element distribution, which is set to

$$
\mu_{i}=\frac{1}{Z_{i}} \sum_{j=1}^{N} \exp \left(-\frac{h_{i j}}{2 \sigma_{d}^{2}}\right) \cdot p_{j}
$$

Based on distribution prior, we can simply remove foreground noises in the image border regions by setting different thresholds for different border sides since their different probabilities of connecting with salient objects. For simplicity, we set an adaptive threshold derived by [14] for bottom side and whose twice for the other sides. Then, the remaining superpixels in the image borders are selected as background prior set as $B G$, which is more stable and reliable than the original four borders. It can remove most of the foreground noises in the image borders

Then, background based saliency map can be formulated by measuring the contrast versus the obtained background prior. However, the superpixels may be distinctive with each other in background prior if the input image is complex. In other words, a superpixel in background regions will only be similar with some part none all of the superpixels in background prior, while a superpixel in foreground regions will be dissimilar with all of the background prior. Thus, it is not a good choice to sum up all the differences together between a superpixel and the background prior to measure its saliency value. To solve it, saliency can be measured in four image borders [12] or three background parts obtained by K-means clustering [13] respectively. Different with them, we only sum up the minimum $L$ differences to measure backgroundness saliency, which is defined as:

$$
B_{i}=\sum_{l=1}^{L} \tilde{h}_{i l}, i=1, \ldots, N
$$

where $\tilde{h}_{i l}$ denotes the ascending sorted histogram distance between superpixel $i$ and superpixel $l$ belong to background prior. Here, $L$ is adaptively set as $L=\frac{|B G|}{10}$, where $|B G|$ is the cardinality of the background prior set BG. Then the coarse saliency map of our proposed distribution guided backgroundness (DGB) can be obtained by normalizing $B$ to $[0,1]$.

\subsection{Refined Saliency map}

After DGB, we get the coarse saliency map. To further improve it, we first use the following sigmoid function to make salient objects more uniformly. Then, we integrate this result with $S$ by linear summation to improve the quality of the saliency map.

$$
\begin{aligned}
& S_{\text {final }}=\frac{1}{2}\left(S+\frac{1}{1+\exp (-\Phi(S))}\right) \\
& \Phi_{i}(S)= \begin{cases}10 \cdot\left(\frac{S_{i}}{T h 2}-1\right), & S_{i}<T h 2 \\
10 \cdot \frac{S_{i}-T h 2}{1-T h 2}, & S_{i} \geq T h 2\end{cases}
\end{aligned}
$$

where $T h 2$ is a threshold to segment $S$ and is obtained by Otsu [14] in this paper. Finally, we smooth the integrated saliency map to produce pixel-wise result by fast bilateral filter [11], which is an edge-preserving smoothing operator and has similar efficiency but better performance than the guided filter used in [15]. The main steps of the proposed saliency detection algorithm are summarized in Fig.2.

\section{Experiment Result}

To measure the performance of proposed method, we 
tested identification using 141 images. On account of no one has yet detected with significant application to do the Aged Home-care in this area, forasmuch we can't get the aged database directly from the network library, therefore the tested source image are download on the internet. At present, only a small number of documents for reference. It is still challenging to develop efficient saliency detection algorithms.
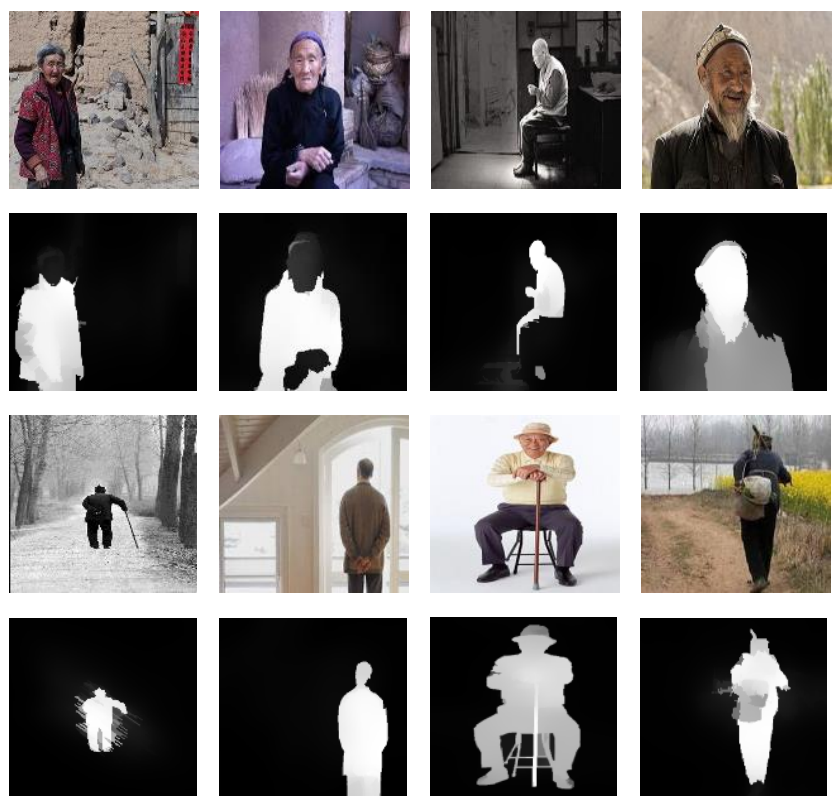

Fig.3.The experiments results of our method, the first and the third line are the input images, the second and the last line are the saliency maps of the input images.
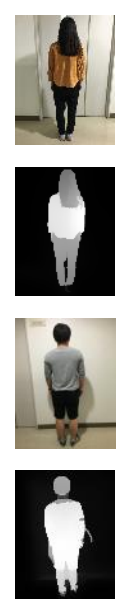

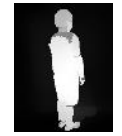

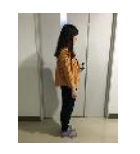
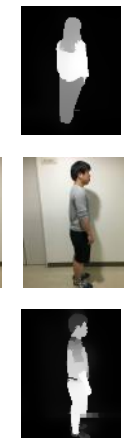
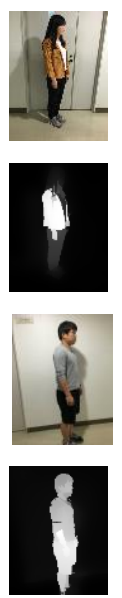
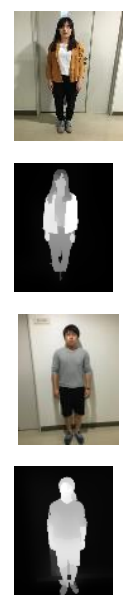
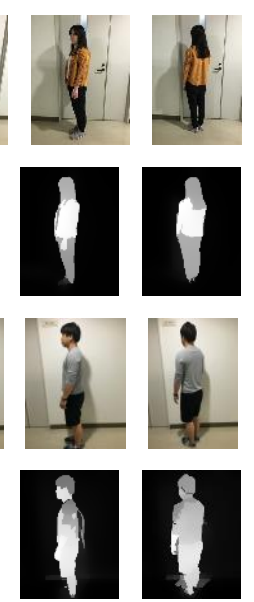

Fig.4.The input images with different angle of the person with different body types. The first line and the third line are the input images, the second line and the last line are the detected results of the input images.
As the results we can see in the Fig.3 the first and the third row are the input images, the second and the last row are the saliency maps of the input images our proposed method could detect the aged easily. Even face the different angle such the back, the front which solved the problem of face recognition technology proposed prior. Besides, we suspect our SPS can outperform in different body types. In order to verify our theory, we need do more test about the poses angles and the body types as we illustrate in the Fig.4. In addition, this model which does not need iteration is more simple and efficient.

\section{Conclusions}

We present a simple and efficient bottom-up saliency detection model. We first propose a discriminative histogram feature metric by combing multiple color space and gradient magnitude channel to handle complex images. Then, we use this metric to compute background based coarse saliency map by introducing distribution as prior to remove foreground noises in the image boundaries. Finally, a simple refinement is applied to generate pixel-wise and smooth saliency maps. Fortunately speaking, the experiment results show that our proposed method can hold the different angles and the different body types. However, how to apply it to the home-care system is still be considered. Therefore, next purpose of the study is connect it to the sensor and use machine learning to analysis the aged behavior that could do more effort to the Aged Homecare.

\section{Acknowledgment}

The authors would like to express their sincere thanks to the anonymous reviewers for their invaluable suggestions and comments to improve this paper. This work was supported by University Science Research Project of Jiangsu Province (15KJB510032), and "Lv Yang Jin Feng" Program of Yangzhou.

\section{References}

(1) A.Borji, D.N.Sihite, and L.Itti: "Salient object detection: A benchmark",In ECCV, 2012.

(2) ITTI L, KOCH C: "Computational modeling of visual attention", In Nature Reviews Neuroscience 2, pp.194-203, 2001. 
(3) Hou, X., Zhang, L.Saliency Detection: “A Spectral Residual Approach", In: CVPR, 2007.

(4) Cheng, M.-M., Zhang, G.-X., Mitra, N. J., Huang, X., $\mathrm{Hu}$, S.-M.: "Global Contrast Based Salient Region Detection",In CVPR, 2011.

(5) Cheng, M.-M., Zhang, Z., Lin, W.-Y., Torr, P.BING: "Binarized Normed Gradients for Objectness Estimation at 300fps", In CVPR, 2014.

(6) U. Rutishauser, D. Walther, C. Koch, and P. Perona, "Is bottom-up attention useful for object recognition?" in CVPR, 2004.

(7) X. Li, Y. Li, C. Shen, A. Dick, and A. Hengel, "Contextual hypergraph modeling for salient object detection," in ICCV, 2013.

(8) V. Mahadevan and N. Vasconcelos, "Saliency-based discriminant tracking," in CVPR, 2009.

(9) L. Itti. Automatic foveation for video compression using a neurobiological model of visual attention. IEEE Trans. Image Proc., vol. 13, no. 10, pp. 1304-1318, Oct 2004.

(10)Song Lu, Vijay Mahadevan, Nuno Vasconcelos. Learning optimal seeds for diffusion-based salient object detection. In CVPR 2014.

(11)Sylvain Paris, Fredo Durand. A Fast Approximation of the Bilateral Filter using a Signal Processing Approach. In ECCV, 2006.

(12)R. Achanta, A. Shaji, K. Smith, A. Lucchi, P. Fua, and S. Susstrunk. Slic superpixels. Technical report, 2010.

(13) Yao Qin, Huchuan Lu, Yiqun $\mathrm{Xu}$ and He Wang. Saliency Detection via Cellular Automata. In CVPR 2015.

(14)N. OTSU. A threshold selection method from gray level histograms. IEEE Trans. Syst. Man Cybern., 9(1) (1979) 62-66.

(15)Jingang Sun, Huchuan Lu, Xiuping Liu, Saliency Region Detection based on Markov Absorption Probabilities, IEEE Transaction on Image Processing, 2015, Vol.24, No.5, pp. 1639-1649. 